\title{
The Mammalian Staufen Protein Localizes to the Somatodendritic Domain of Cultured Hippocampal Neurons: Implications for Its Involvement in mRNA Transport
}

\author{
Michael A. Kiebler, ${ }^{1}$ Indradeo Hemraj, ${ }^{1}$ Paul Verkade, ${ }^{1}$ Martin Köhrmann, ${ }^{1}$ Puri Fortes, ${ }^{2,3}$ Rosa M. Marión, ${ }^{3}$ \\ Juan Ortín, ${ }^{3}$ and Carlos G. Dotti ${ }^{1}$ \\ European Molecular Biology Laboratory, ${ }^{1}$ Cell Biology Programme and ${ }^{2}$ Gene Expression Programme, 69012 Heidelberg, \\ Germany, and ${ }^{3}$ Centro Nacional de Biotecnologia, Consejo Superior de Investigaciones Científicas, Campus de \\ Cantoblanco, 28049 Madrid, Spain
}

In hippocampal neurons, certain mRNAs have been found in dendrites (Steward, 1997), and their localization and translation have been implicated in synaptic plasticity (Martin et al., 1997). One attractive candidate to achieve transport of mRNAs into dendrites is Staufen (Stau), a double-stranded RNA-binding protein, which plays a pivotal role in mRNA transport, localization, and translation in Drosophila (St. Johnston, 1995). Using antibodies raised against a peptide located in the RNA-binding domain Ila and a polyclonal antibody raised against a recently cloned human Staufen homolog, we identify a $65 \mathrm{kDa}$ rat homolog in cultured rat hippocampal neurons. In agreement with the exclusive somatodendritic localization of mRNAs in these cells, we find that Staufen is restricted to the same

One of the mechanisms to generate cell polarity is the intracellular localization of mRNAs to discrete subcellular locations. In differentiating myoblasts and embryonic fibroblasts, for example, $\beta$-actin message localizes to the leading lamellae at the cell periphery (Lawrence and Singer, 1986; Hill and Gunning, 1993; Kislauskis et al., 1993). In the Drosophila oocyte, bicoid, oskar, and gurken, mRNAs are targeted to three distinct positions within the cell, determining the polarity of the anteroposterior and dorsoventral axes of the embryo (St. Johnston, 1995). In the Xenopus laevis oocyte, at least three different compartments contain localized messages, such as Vg1 (St. Johnston, 1995). In oligodendrocytes, the message for myelin basic protein has been shown to be transported into processes (Ainger et al., 1993, 1997). In the mammalian nervous system, several distinct polarized nerve cells sort mRNAs coding for cytoskeletal, kinase, or $\mathrm{Ca}^{2+}$ dependent proteins to their respective dendrites (Steward, 1997). Although the precise function of mRNA localization in dendrites is still unclear, it has been hypothesized that it offers the possi-

\footnotetext{
Received Sept. 9, 1998; accepted Oct. 15, 1998.

This work was supported by a postdoctoral fellowship from the Deutsche Forschungsgemeinschaft (M.A.K.), research fellowships from the HFSPO (M.A.K. and P.F.), a University Scholar Program of the University of Pennsylvania (I.H.), an EC grant and fellowship (P.V. and P.F., respectively), the PNFPI (R.M.M.), PGC Grant PB94-1542 (J.O.), and Sonderforschungsbereich Grant SFB-317 (C.G.D.). We thank B. Hellias and S. Brendel for technical assistance, Drs. L. Des Groseillers and D. St. Johnston for courteously communicating unpublished results and support, Drs. S. Cohen, A. Ephrussi, N. Gunkel, I. Mattaj, J. C. López-García, P. Scheiffele, and D. St. Johnston for discussions and/or critical reading of this manuscript.

Correspondence should be addressed to M. Kiebler, European Molecular Biology Laboratory, Cell Biology Programme, Meyerhofstrasse 1, D-69117 Heidelberg, Germany. E-mail address: Kiebler@EMBL-Heidelberg.de

Copyright (C) 1998 Society for Neuroscience $\quad 0270-6474 / 98 / 190288-10 \$ 05.00 / 0$
}

domain. By immunoelectron microscopy, we show enrichment of the mammalian homolog of Stau (mStau) in the vicinity of smooth endoplasmic reticulum and microtubules near synaptic contacts. Finally, the association of the mStau with neuronal mRNAs is suggested by the colocalization with ribonucleoprotein particles specifically in distal dendrites known to contain mRNA, ribosomes, and translation factors (Knowles et al., 1996). These results suggest a role for mStau in the polarized transport and localization of mRNAs in mammalian neurons.

Key words: mammalian Staufen; double-stranded mRNAbinding protein; hippocampal neurons; mRNA transport; ribonucleoprotein particles; SYTO14

bility of local translational control after synaptic activation, thereby modifying the existing synaptic strength (Martin et al., 1997). To test this hypothesis, it appears essential to first identify the molecular machinery involved in targeting such messages to the synapse. One promising candidate is a double-stranded RNAbinding protein called Staufen (Stau). In invertebrates, Stau is required for the proper localization of maternal mRNAs to either the anterior or the posterior pole of the Drosophila oocyte and in the asymmetric localization of mRNAs, such as prospero in Drosophila neuroblasts (St. Johnston, 1995; Campos-Ortega, 1997; Li et al., 1997; Broadus et al., 1998). Most notably, Stau is also involved in the translation of oskar message at the posterior pole (Breitwieser et al., 1996). Finally, Stau is found in ribonucleoprotein particles (RNPs), which are then actively transported along the microtubules (MTs) (Ferrandon et al., 1994).

Taking advantage of published expressed sequence tag (EST) sequences [e.g., AA106767] from mouse and human with significant homology to Stau, we raised our own specific antibodies and studied the pattern of expression and distribution of the mammalian Stau (mStau) in rat hippocampal neurons in culture. Furthermore, we used a specific antibody against the C-terminal domain of human Stau, recently characterized in HeLa cells (Marión et al., 1999).

\section{MATERIALS AND METHODS}

Materials. The following antibodies were used in the indicated dilutions: monoclonal antibody against MAP2 (Sigma, St. Louis, MO) at 1:500 (De Hoop et al., 1994); the monoclonal antibody against tau-1 (Boehringer Mannheim, Mannheim, Germany) at 1:200 or 1:500 (Binder et al., 1985); and the monoclonal antibody against ribophorin I (courteously provided by D. Meyer, University of California at Los Angeles) at 1:100 (Hortsch 


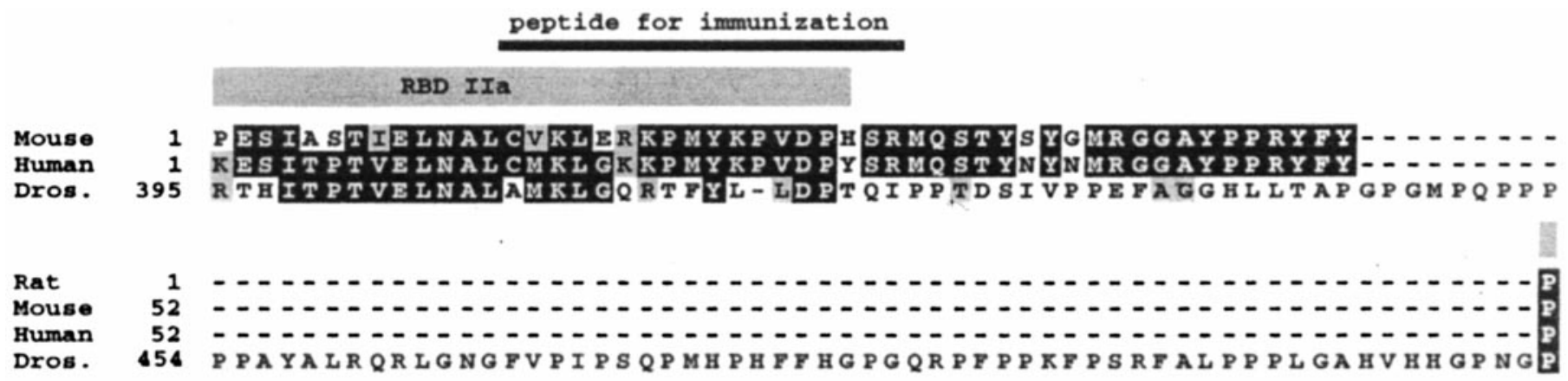

\section{RBD IIb}

Rat Mouse Human Dros.

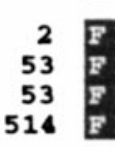

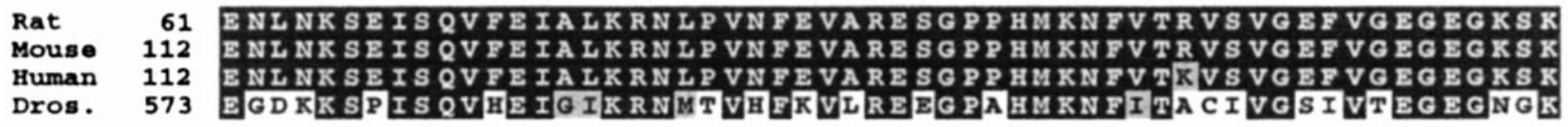

Figure 1. Conserved domain structure in mStau protein. An amino acid alignment from rStau (EMBL accession number AJ10200), mouse (EST clones AA106767 and AA896810), human (EST clones AA206573 and R62169) and Drosophila (M69111) is shown. The partial rStau cDNA was cloned by PCR, and its sequence was deposited into EMBL database (EMBL accession number AJ10200). Over the entire 117 amino acid domain, rStau shows $48 \%$ identity (71\% similarity) compared with the Drosophila sequence. The recent identification of both a human Stau (Marión et al., 1999) and a mouse fulllength Stau clone (Wickham et al., 1999) with high homologies to Drosophila Staufen protein verified the sequences predicted from the EST clones. Residues shaded in black and gray are regions of identity and similarity, respectively. The gray bars indicate the three conserved RBDs, IIa, IIb, and III; the black bar indicates the peptide chosen for immunization within the highly homologous RBD IIa region of Staufen protein.

et al., 1986). As secondary antibodies, a rhodamine-conjugated goat anti-rabbit whole Ig (1:100; 55674; Organon Teknika-Cappel, Durham, NC), a Cy5-conjugated donkey anti-rabbit whole Ig (1:300; 711175 152; Dianova, Hamburg, Germany) or FITC-conjugated sheep anti-mouse whole Ig (1:50; N1031; Amersham, Arlington Heights, IL) were used.

Cloning of the rat Staufen homolog. Two PCR primers were designed based on the sequence alignment (Fig. 1): sense $\left(5^{\prime} \rightarrow 3^{\prime}\right)$ TACTTTTAC CCATTTCCAGT; and antisense: ATCTTCTTGCTTTTCCCTTC. A PCR was performed with these primers on a rat embryonic $\lambda \mathrm{gt} 11$ library (a generous gift from Drs. H. Monyer and P. Seeburg, ZMBH, University of Heidelberg, Heidelberg, Germany), and the resulting $370 \mathrm{nt}$ fragment was cloned into the pGEM-T vector (Promega, Madison, WI). Both strands were sequenced, and the resulting cDNA sequence was deposited into the European Molecular Biology Laboratory (EMBL) database (accession number AJ10200).

Cell culture, live labeling, and stimulation. Primary hippocampal neurons derived from rat embryos were cultured according to the protocol of Goslin and Banker (1991) and De Hoop et al. (1998). SYTO14 labeling $(50 \mathrm{nM})$ of cultures was essentially performed as described previously (Knowles et al., 1996), with the following modification. To ensure high uptake of SYTO14 into the cells, hippocampal neurons were stimulated by membrane depolarization to induce neurotransmitter release (Rosa et al., 1985; Parton et al., 1992). In brief, cells were incubated in SYTO14containing depolarizing solution (in mM: $97 \mathrm{NaCl}, 35 \mathrm{KCl}, 10 \mathrm{HEPES}$, pH 7.35, 2.2 $\mathrm{CaCl}_{2}, 0.33 \mathrm{Na}_{2} \mathrm{HPO}_{4}, 0.44 \mathrm{KH}_{2} \mathrm{PO}_{4}, 4.2 \mathrm{NaHCO}_{3}$, and 5.6 glucose) for $5 \mathrm{~min}$ at $37^{\circ} \mathrm{C}$, transferred into SYTO14-containing normal medium for $5 \mathrm{~min}$, and finally into normal medium for $25 \mathrm{~min}$ at $37^{\circ} \mathrm{C}$. Neurons were briefly rinsed in HBSS and then fixed.

Antibodies and Western blots. The expression of the His-tagged C-terminal part of human Stau (pRSTL) and the production of polyclonal antibodies against the purified protein from Escherichia coli were described elsewhere (Marión et al., 1999). Best results with the antiserum were obtained with a dilution of 1:200 for immunofluorescence. As controls for antibody specificity, the primary antibody was omitted or the corresponding preimmune serum was used (data not shown). Furthermore, the antisera was preincubated with the purified, bacterially overexpressed antigen $(10 \mu \mathrm{g} / \mathrm{ml})$ for $1 \mathrm{hr}$ at $4^{\circ} \mathrm{C}$ and then directly used for immunofluorescence as described above. Virtually all the dendritic label was abolished (data not shown).

Two additional polyclonal antibodies were raised against a synthetic peptide derived from the mouse Stau sequence (EST clone AA106767) in the dsRNA-binding motif IIa (CVKLERKPMYKPVDPHSR) coupled to KLH. These antisera were monospecific for rat Stau (rStau) (Fig. 1 , lanes 5 and 6 ). Best results with both antisera were obtained with a dilution of 1:500 or higher for Western blots or 1:200 for immunofluorescence. Neuronal and glial extracts were prepared as described previously (De Hoop et al., 1998). To ensure sharp bands for SDS-PAGE, lipids were extracted with chloroform-methanol (Wessel and Flügge, 1984) before SDS-PAGE.

Immunocytochemistry was performed as described by Bradke and Dotti (1997). In brief, neurons grown on glass coverslips were fixed in 4\% paraformaldehyde (PFA), quenched with ammonium chloride, permeabilized with Triton X-100, and incubated with the respective antibodies. Finally, cells were mounted with Mowiol and DABCO as described.

Image analysis and quantitation. Fluorescent microscopy was performed with a Zeiss (Oberkochen, Germany) Axioskop using either a $40 \times, 63 \times$, or $100 \times$ objective, standard FITC, rhodamine, or Texas Red filters, and a $100 \mathrm{~W}$ mercury arc lamp. Confocal microscopy was performed with a Leica (Nussloch, Germany) DM IRBE confocal microscope using either a $40 \times$ or $63 \times$ objective, and standard FITC, rhodamine, or Cy5 filters. Because excitation of SYTO14 led to emission in the FITC, rhodamine, and even Texas Red channel (data not shown; Knowles et al., 1996), Stau was detected with a Cy5-conjugated secondary antibody. For fluorescence microscopy, images were acquired using a cooled CCD camera (Photonic Science) controlled by the IP-Lab (Signal Analytics) or Open Lab version 1.7.6 (Improvision) software packages and assembled using Adobe photoshop versions 3.0.4. or 4.0. For confo- 
cal microscopy, images were acquired using TCS NT (version 1.5; Leica), imported into NIH Image, and assembled.

Electron microscopy. Cultured rat hippocampal neurons (5-week- old) grown on glass coverslips were briefly rinsed in prewarmed sodium cacodylate buffer (CB) (100 mM, pH 7.4) and immediately fixed in $4 \%$ PFA and $0.05 \%$ glutaraldehyde in CB for $10 \mathrm{~min}$ at room temperature (RT). All solutions were made in tridistilled water. The procedure described is based on a preembedment protocol (Van Lookeren Campagne et al., 1992) in which $5 \mathrm{~nm}$ gold particles in CB can penetrate the plasma membrane without previous permeabilization. The small gold protein A complex ensured significant labeling intensity, with good ultrastructure after embedment into Epon. After fixation, the cells were rinsed in blocking buffer (BB) (CB including 5\% fetal calf serum and $0.2 \%$ cold water fish skin gelatin), and free aldehydes were quenched for 10 min with $0.05 \% \mathrm{NaBH}_{4}$ and $200 \mathrm{~mm}$ glycine in CB. The coverslips were again extensively rinsed with $\mathrm{BB}$, and immunolabeling was performed with anti-human mStau polyclonal antibody (1:200 in BB) overnight at $4^{\circ} \mathrm{C}$. After extensive rinsing in $\mathrm{BB}$, the coverslips were incubated with $5 \mathrm{~nm}$ gold particles coupled to protein A (purchased from J. Slot, Utrecht, The Netherlands) for $1 \mathrm{hr}$ at RT, and then cells were extensively rinsed in $\mathrm{BB}$ and tridistilled water. Cells were processed according to routine Epon-embedding procedures. Ultrathin sections were cut parallel to the cells, counterstained with uranyl acetate and lead citrate, and viewed under a Zeiss electron microscope. In controls, omitting the primary antibody, no gold particles were detected (data not shown). Figure $5 a$ was processed using Adobe Photoshop 4.0 to enhance visibility of gold particles.

\section{RESULTS}

\section{rStau is a $65 \mathrm{kDa}$ protein}

We wanted to explore whether a putative mammalian homolog of Stau is present in neurons, and moreover, whether it may play a role in mRNA localization in hippocampal neurons. For this reason, we searched the EMBL database with the Drosophila Stau sequence and identified several EST clones from both human and mouse with high homology to Drosophila Stau (Fig. 1). To confirm the presence of a mStau homolog in rat as suggested by the presence of EST clones, we cloned a C-terminal fragment of rStau cDNA by PCR and deposited its sequence into the EMBL database (accession number AJ10200). A sequence alignment with the Drosophila sequence (Fig. 1) reveals a high degree of homology; rStau shows $48 \%$ identity ( $71 \%$ similarity) compared with the Drosophila sequence over the entire 117 amino acid domain. A comparison with the mentioned EST sequences shows that rStau is 92 and $98 \%$ identical (96 and 99\% similar) to human and mouse Stau, respectively. Interestingly, only two amino acids differ from the corresponding mouse sequence. The recent identification of both a human Stau (Marión et al., 1999) and a mouse full-length Stau clone (Wickham et al., 1999) with high homologies to the Drosophila Stau protein demonstrated that the sequence derived from the EST clones were real and further substantiated our findings that mStau homologs exist.

Based on the sequence comparisons, we chose a conserved peptide in the RNA-binding domain (RBD) IIa region of Stau protein (Fig. 1) for immunization of rabbits. The epitope is well conserved in mStau homologs (the mouse epitope is $78 \%$ identical and $89 \%$ similar to human Stau) and still shows some homology to the corresponding Drosophila region (39\% identity and $50 \%$ similarity). It is important to note that this peptide did not show any significant homology to any other proteins in the database. The immunization yielded polyclonal antibodies specific for mStau. Additionally, we took advantage of a specific antibody against the C-terminal domain of human Stau, recently characterized in HeLa cells (Marión et al., 1999).

To determine the molecular weight of the rat homolog, we performed Western blotting on hippocampal extracts. The two

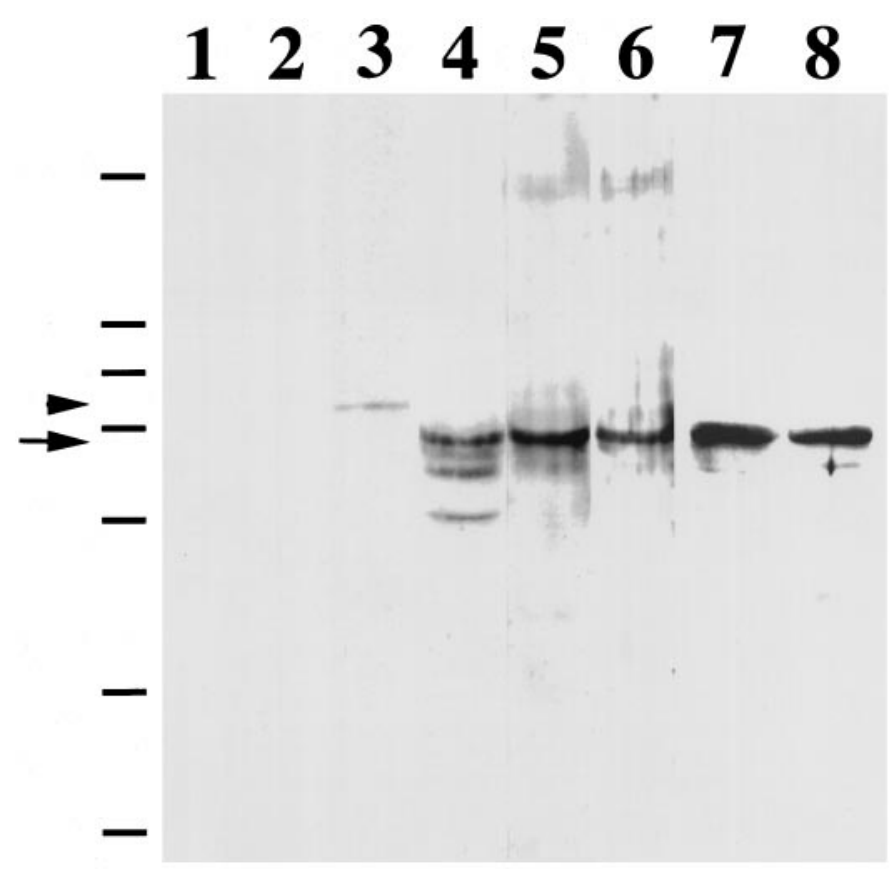

Figure 2. rStau is a $65 \mathrm{kDa}$ protein. Western blot of rat hippocampal neuronal extracts probed with three different anti-mStau antibodies (lanes 4-6) and its corresponding preimmune antisera (lanes 1-3). Two of the three antisera were monospecific for the $65 \mathrm{kDa}$ band (arrow). The only visible band from one of the preimmune sera was a $72 \mathrm{kDa}$ band in lane 3 (arrowhead). When we compared the expression level of Stau on astrocyte (lane 7) and hippocampal neuronal extracts (lane 8), we found approximately the same signal for mStau. The size of the molecular weight markers is indicated on the left: 202, 104, 82, 66, 48, 33, and 28 $\mathrm{kDa}$. All three antisera gave identical patterns of labeling (data not shown) as the one used in Figures 3 and 4.

independent anti-rStau peptide antibodies, as well as the antihuman Stau antisera, recognized a $65 \mathrm{kDa}$ protein in rat neuronal extracts (Fig. 2, lanes 4-6), whereas the corresponding preimmune antisera did not (Fig. 2, lanes 1-3). This is in agreement with the predicted molecular weight of the full-length human Stau gene product and its apparent electrophoretic mobility (Wickham et al., 1999). This indicates that all our antibodies recognize the same rStau homolog. On Western blots from both astrocyte (Fig. 2, lane 7) and hippocampal extracts (lane 8), we found approximately the same amount of expression of mStau, indicating that mStau is expressed in both cell types. When we tested the two anti-peptide antibodies on Western blots from HeLa and mouse hippocampal extracts, we also detected the same band (data not shown). Together, these data indicate that all our antibodies are monospecific for a $65 \mathrm{kDa}$ protein and that this represents the rStau homolog.

\section{Expression pattern of Staufen during development of hippocampal neurons}

We then went on to examine the expression pattern of mStau during neuronal development in hippocampal neurons in culture; several morphologically distinct events have been characterized leading to polarization of these cells (Dotti et al., 1988). During early stages of development (stages 1-3), when polarization of neurites into axons and dendrites has not yet been achieved, $\mathrm{mStau}$ is present in all processes (Fig. 3a,c,e). $\alpha$-Tubulin immunoreactivity of the same cell served as a cytoskeletal marker to 

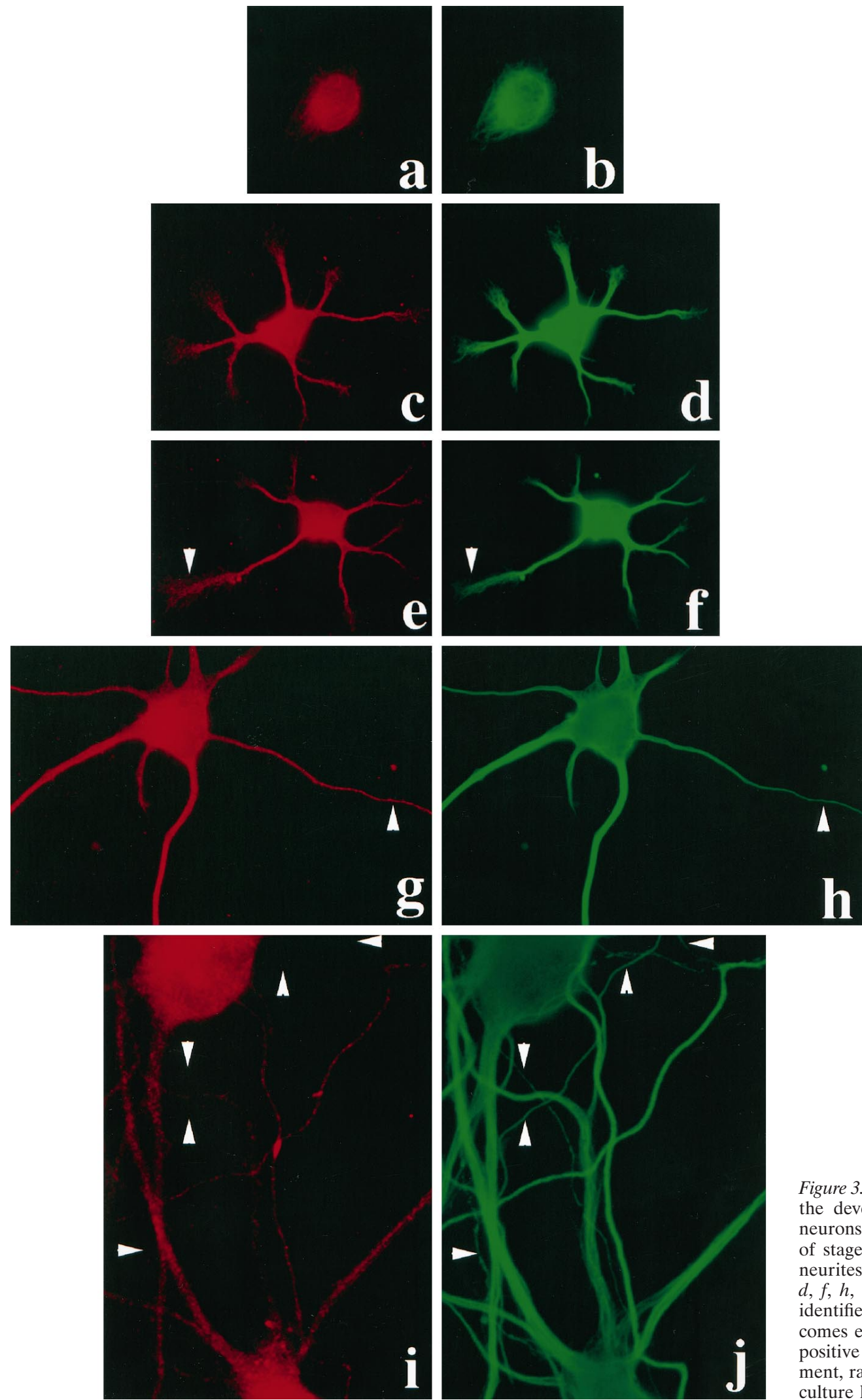

Figure 3. Intracellular distribution of rStau during the development of polarity in rat hippocampal neurons. mStau labeling is present in the cell body of stage 1 cells $(a)$ and in the cell body and all neurites of stage 2,3 , and 4 ( $c, e, g$, respectively). $b$, $d, f, h$, and $j$ show all the processes of the cells identified by $\alpha$-tubulin. At stage $5(i)$, mStau becomes excluded from some of the thin, $\alpha$-tubulinpositive processes ( $j$, arrowheads). In this experiment, rat hippocampal neurons for 2,4 , and $8 \mathrm{~d}$ in culture have been used.

label all processes (Fig. 3b,d,f). The presence of mStau in all processes is still evident in stage 4 cells (Fig. $3 g, h$ ). However, mStau becomes excluded from some of the processes (Fig. 3ij, at stage 5 neurons. Given that the mStau-negative processes have an axon-like morphology (thin uniform diameter; see Fig. 5) and that mRNA transport in these cells occurs in dendrites but not axons, it appears that the mStau-positive processes are dendrites. This was investigated next. 


\section{Staufen is expressed in the somatodendritic domain of hippocampal neurons}

In fully polarized hippocampal neurons, axons and dendrites can be distinguished by morphological, immunological, and functional criteria; also, numerous mRNAs specifically localize to dendrites (Craig and Banker, 1994; Steward, 1997). In a representative mature hippocampal neuron (Fig. 4a), mStau immunoreactivity was present in cell bodies, as well as in dendrites (Fig. $4 b$, arrows). MAP2 immunoreactivity of the same cell served as a marker to identify dendrites (Fig. 4c) (Cáceres et al., 1984). mStau was not found in axons (Fig. 4c, arrowheads, MAP2negative processes). Also, mStau protein was not found in tau-1labeled axons (Fig. 4d-f) (Binder et al., 1985). Because mStau was expressed in immature axons (Fig. $3 g, h$ ) but not in fully mature axons (Fig. 3i,j), the size of axons cannot be the factor preventing the detection of mStau. Additionally, we further investigate this fact using electron microscopy (EM) (Fig. 5).

As controls for antibody specificity, the primary antibody was omitted, the corresponding preimmune serum was used, or the $\alpha$-Stau antisera was preincubated with its antigen before immunolabeling. All of these treatments virtually abolished any specific staining for Stau (data not shown). All three antibodies, when tested by immunofluorescence microscopy, gave a similar pattern of labeling as described above (data not shown). Together with the results of the Staufen localization at earlier stages, the distribution of Staufen corresponds to that of mRNAs: it is present in all neurites at earlier stages and becomes preferentially restricted to the somatodendritic region in fully mature neurons.

\section{Staufen is found to be associated with tubular structures and MTs in dendrites}

To confirm the dendrite-specific labeling for mStau obtained by immunofluorescence and to fully characterize its intracellular location, we performed preembedment immunoelectron microscopy (Van Lookeren Campagne et al., 1992). In Figure 5, $a$ and $b$, representative synapses are shown in which mStau (arrowheads) is almost exclusively found in dendrites but not in axon terminals. When we performed quantification of gold particles (Table 1) in 10 axons and dendrites each, we found $1.0 \pm 0.4$ (mean \pm SEM) gold particles in axons compared with $13.4 \pm 1.5$ (mean \pm SEM) in dendrites. This represents $94 \%$ postsynaptic mStau. Some dendritic label was in close proximity to tubular structures, most likely representing endoplasmic reticulum (ER). This association was found in $38 \pm 4.5 \%$ (mean \pm SEM) of all gold particles. Synaptic vesicles, multivesicular bodies, and mitochondria were not labeled. In Figure $5 c$, a hippocampal axon is shown fasciculating on a dendrite. The thickening of the axon on the right side of Figure $5 c$ represents a varicosity forming a synapse toward the underlying dendrite. As before, mStau label was only observed in the dendrite but not in the axon, confirming the fluorescence data of Figure 4. Another interesting piece of information from our EM studies was the presence of abundant gold particles close to MTs. When we examined all the EM micrographs with clearly identifiable MTs, we found 30 of 32 gold particles closer than $18 \mathrm{~nm}$ (Verkade et al., 1997) to an MT (96 \pm $4.2 \%$, mean \pm SEM), strongly arguing in favor of an mStau-MT association. This is of particular interest, because the mStau protein contains an MT-binding domain similar to that of MAP1B, and it seems to bind MTs in vitro (Wickham et al., 1999). Together, these data show that mStau is specifically found in dendrites, but not axons, of neurons in which it concentrates in the vicinity of ER and MTs.

\section{Staufen colocalizes with ER}

To highlight the organelle-bound form of mStau, we removed the nonmembrane-bound $\mathrm{mStau}$ with the nonionic detergent saponin and analyzed its distribution by immunofluorescence. Under this condition, mStau labeling appeared in the form of prominent, large clusters in dendrites (Fig. $6 a$ ). Given the abundance of ER cisternae in dendrites, at least part of these clusters of mStau could represent ER structures as already suggested by EM. Because mStau may be involved in transport of certain mRNAs and potentially in their translation, we next studied its ER-association using the rough ER marker ribophorin I (Hortsch et al., 1986) (Fig. $6 c, e$ ). Indeed, mStau (Fig. $6 b, d$ ) showed a high degree of colocalization with ribophorin I, confirming that mStau is associated directly with either ER membranes or ribosomes attached to rough ER. This is in good agreement with data in HeLa cells in which mStau was found to (1) colocalize with rough ER and (2) cosediment with polysomes (Marión et al., 1999) (data not shown). However, mStau did not show any significant colocalization with the trans-Golgi network marker TGN38 (Lucio et al., 1990). In this context, it is particularly interesting to note that, in Xenopus, the ER itself has been implicated in the localization of the mRNA Vg1 in an MT-dependent step with the help of the ER-associated protein Vera (Deshler et al., 1997).

\section{Staufen is found in RNPs containing RNA}

The membrane-permeable nucleic acid dye SYTO14 stains intracellular granules in living hippocampal neurons, which contain poly $\left(\mathrm{A}^{+}\right)$mRNA, the 60S ribosomal subunit, and the elongation factor $1 \alpha$, and which move in an MT-dependent manner (Knowles et al., 1996). Hence, we used SYTO14 to fluorescently label the described granules and to test whether mStau is present in these large RNPs in dendrites. Because SYTO14 also labels mitochondria (Knowles et al., 1996), additional label will be expected. For higher precision and more restricted labeling, we used confocal microscopy. Figure 7, $a$ and $b$, shows lowmagnification photographs of mStau-SYTO14 colabeling. Some, but not all, of the SYTO14-labeled processes are mStau-positive (Fig. 7a,b, arrows). Because mStau is restricted to dendrites (Figs. 2, 4), Staufen-negative processes correspond to axons (Fig. 7a,b, arrowheads). Figure 7, $c$ and $d$, show a high-magnification view of a branched neuronal dendritic process that contains mStau- and SYTO14-labeled structures. The uppermost process shows almost perfect colocalization (Fig. 7c,d, arrowheads) between SYTO14fluorescent granules and mStau, indicating that mStau is indeed in a complex with RNPs containing mRNAs and ribosomes. We also observed SYTO14-labeled clusters that are mStau-negative. Because mStau did not label mitochondria at both the light microscopical (data not shown) and the electron microscopical level (Fig. 5), these clusters most likely represent mitochondria. In conclusion, the colocalization of mStau with RNPs in dendrites of hippocampal neurons suggest that mStau may be in direct physical contact with dendritic mRNAs contained in these RNPs.

Knowles et al. (1996) provided EM data from cortical neurons in culture showing polyribosomes in large SYTO14-positive granules, and they suggested that these granules may represent a translational unit. In our experimental system, we have not been able to find any Stau-positive polyribosome-containing granules in neurons under our conditions (Fig. 5). It remains to be seen whether or not the large structures double-labeled for Stau and SYTO14 also contain polyribosomes. It is possible that these RNPs represent active transport units that deliver mRNAs to the 

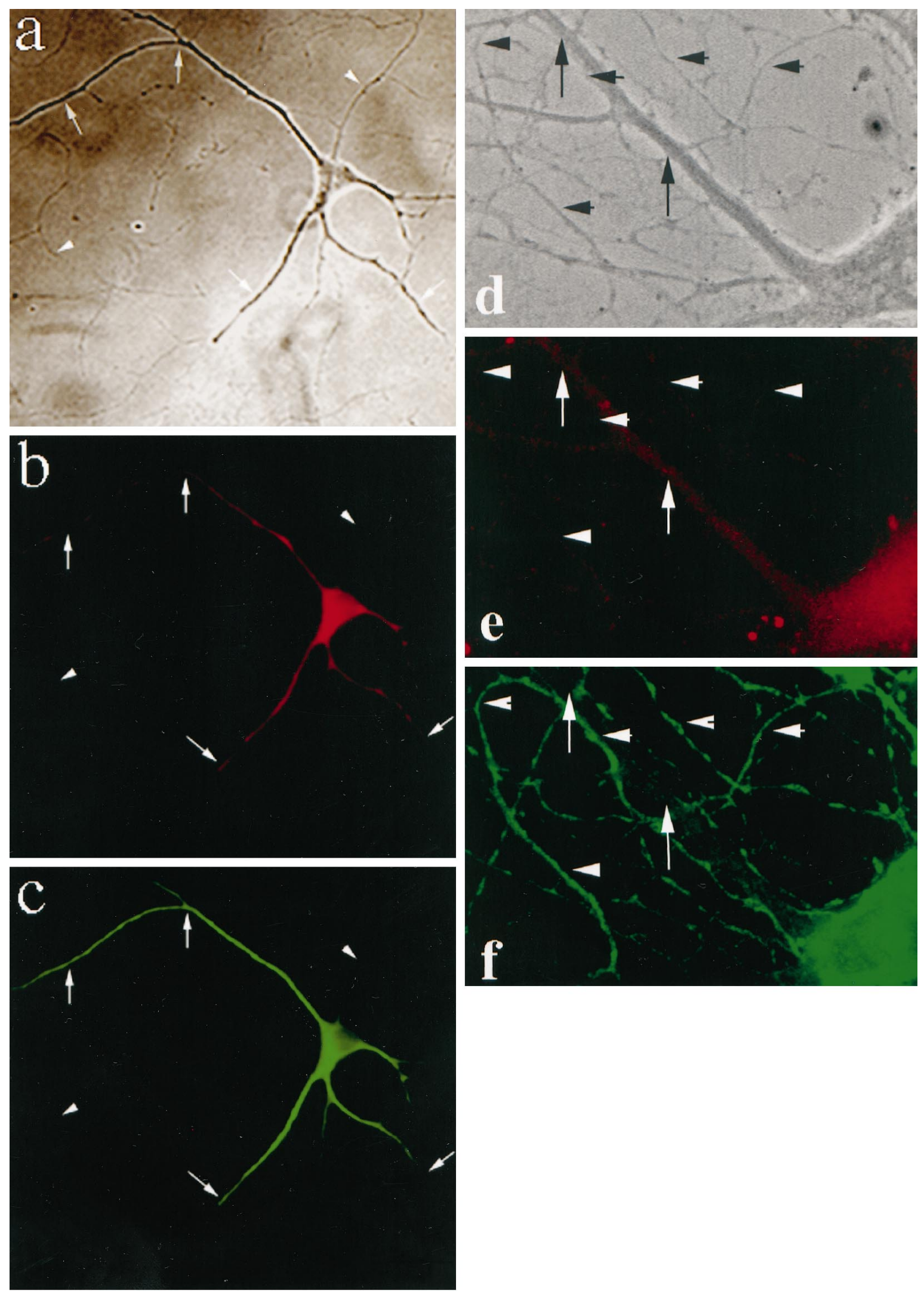

Figure 4. Intracellular distribution of mStau in mature rat hippocampal neurons in culture $(14 \mathrm{~d}$ in vitro $)$. $a$, Phase-contrast image of a representative cell. $b$, mStau labeling is present in the cell body and some of the long processes identified as dendrites with the specific dendritic marker MAP2 ( $c$ ). Note that axons (arrowheads, MAP2-negative processes) are not labeled with the mStau antibody. $d$, Phase-contrast image of another representative cell. Double-immunofluorescence of neurons with mStau $(e)$ and the axonal marker tau-1 $(f)$. Note that axons (arrowheads, tau-1-positive processes) are not labeled with the mStau antibody (arrows, a Stau-positive dendrite). The average diameter of a CA1 pyramidal neuron is between 8 and $12 \mu \mathrm{m}$. 

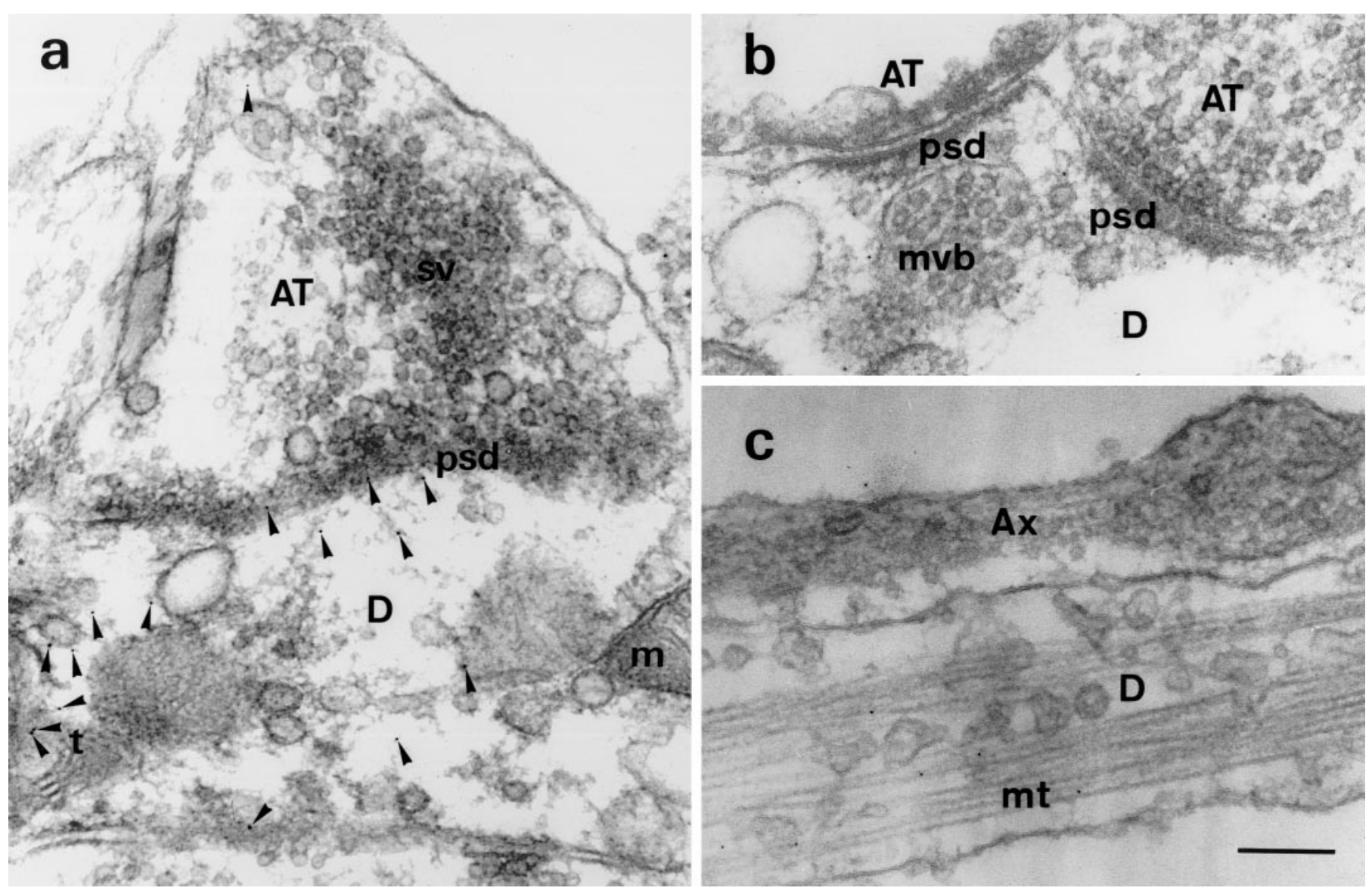

Figure 5. Postsynaptic localization of Staufen protein and association with MTs and ERs. $a$, Staufen gold labeling (arrowheads) is exclusively found in dendrites $(D)$ but not axonal terminals $(A T)$ of hippocampal neurons (ratio of 13.4:1; see Results). Some gold clusters were associated with or in close vicinity of tubular ER structures in the dendritic process ( $38 \%$ of all gold; see Results). $b$, At higher magnification, mStau gold labeling is clearly seen in dendrites but not axonal terminals. $c$, Staufen labeling was found on bundles of MTs $(m t)$ derived from a dendritic process. The nearby fasciculating axon above the dendrite did not contain any mStau label. Scale bar (in $c): a, 250 \mathrm{~nm} ; b, c, 200 \mathrm{~nm}$. $m$, Mitochondria; $s v$, synaptic vesicle; $t$, tubular network; psd, postsynaptic density; $m v b$, multivesicular body.

\section{Table 1. Quantitation of Staufen labeling in hippocampal neurons}

\begin{tabular}{lcc} 
Compartment & $\begin{array}{l}\text { \# of gold particles } \\
\text { (mean } \pm \text { SEM) }\end{array}$ & $\begin{array}{l}\% \text { of gold particles } \\
\text { (mean } \pm \text { SEM) }\end{array}$ \\
\hline Dendrites & $13.4 \pm 1.5$ & $94 \pm 2 \%$ \\
Axons & $1.0 \pm 0.4$ & $6 \pm 2 \%$ \\
\hline
\end{tabular}

Staufen association with

$\begin{array}{lll}\text { Intracellular membranes } & 52 \text { of } 134 & 38 \pm 4.5 \% \\ \text { Microtubules } & 30 \text { of } 32 & 96 \pm 4.2 \%\end{array}$

Random electron micrographs were chosen for this quantitation. In the first row, gold particles within 10 axons and dendrites were counted and expressed as average numbers of gold particles. In the second row, numbers of gold particles were listed that were found to be associated with intracellular membranes (most likely ERs) and MTs.

synapse in neurons and that polyribosomes are rather independent organelles (Steward and Levy, 1982).

\section{DISCUSSION}

Our results demonstrate that mStau is found in the cell body and dendrites of hippocampal neurons in which it may associate with MTs and RNPs at synaptic contacts (Fig. 5). What role could mStau, a double-stranded RNA-binding protein, play in neurons? Segregating mRNAs is a general mechanism to localize protein synthesis to the site of its function in which it can be locally assembled into macromolecular complexes. One striking example of asymmetric mRNA localization occurs in the invertebrate Drosophila oocyte in which Stau is crucial for the proper localization of maternal mRNAs bicoid and oskar to the anterior or the posterior pole, respectively (St. Johnston, 1995). A $400 \mathrm{nt}$ region of the bicoid $3^{\prime}$-UTR has been identified that can bind Stau (Ferrandon et al., 1994). More recently, Stau (in a complex with inscuteable) was shown to be involved in asymmetric mRNA localization in Drosophila neuroblasts, which are progenitor cells of the CNS (Campos-Ortega, 1997; Li et al., 1997; Broadus et al., 1998). These data strongly suggest that mStau may also be involved in dendritic mRNA transport in mammalian neurons. What is the experimental evidence that there is directed intracellular mRNA transport in neurons? First, it has been shown by in situ hybridization that a subset of mRNAs coding for kinases, substrates for kinases, or cytoskeletal proteins exist in dendrites (Steward, 1997). Second, poly $\left(\mathrm{A}^{+}\right)$RNA visualized in RNPs has been identified that move in an MT-dependent manner (Knowles et al., 1996). Third, the presence of organelles of the protein biosynthetic machinery such as ER cisternae and polyribosomes has been identified in dendrites, but not axons of hippocampal neurons (Knowles et al., 1996). Fourth, data suggest that these dendritically transported mRNAs may be translated after being 

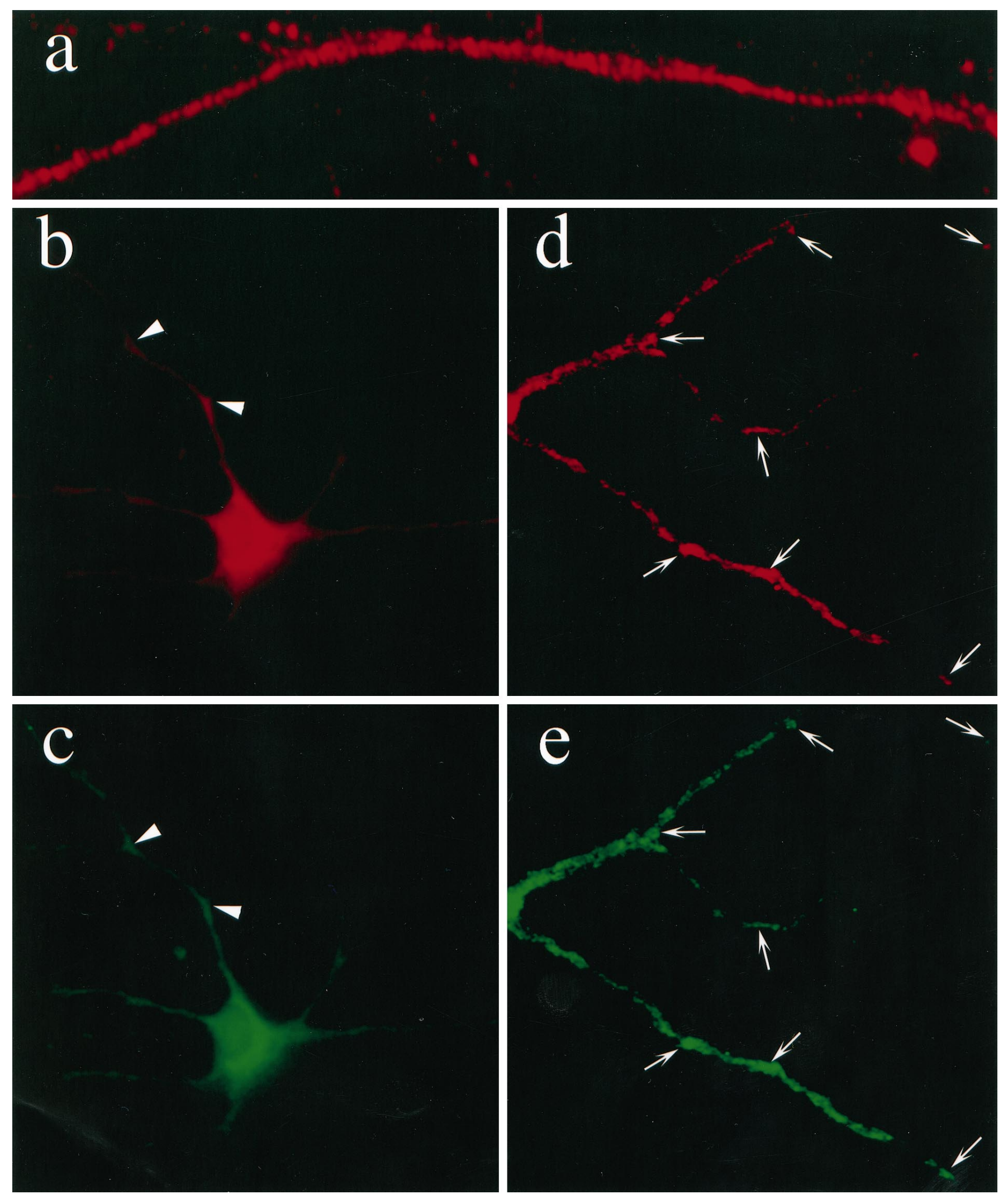

Figure 6. Staufen protein is found in large dendritic clusters and colocalizes with rough ER in mature rat hippocampal cells in culture. Immunofluorescence of a saponin-treated cell $(a)$ using anti-mStau antibodies shows mStau in the form of clusters (most likely membrane-bound). $b-e$, Double-immunofluorescence of neurons with mStau $(b, d)$ and the rough ER marker ribophorin I $(c, e)$ reveals good colocalization between these two proteins (arrowheads and arrows). This confirms data shown in Figure $3 a$ and data obtained in HeLa cells (Marión et al., 1999). 

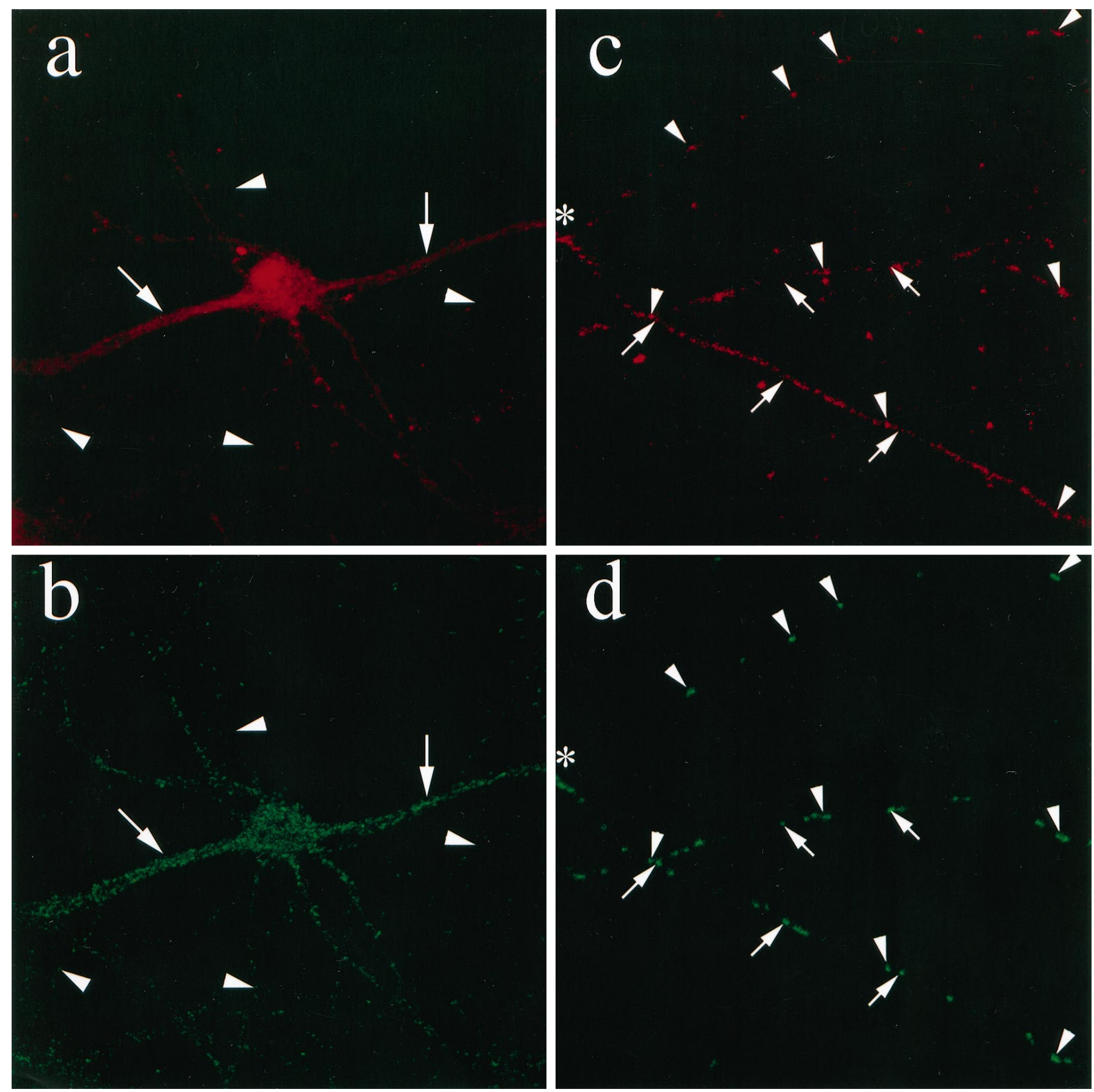

Figure 7. Colocalization of mStau with RNPs in hippocampal neurons. RNPs and mitochondria were visualized with the RNA-specific dye SYTO14 and the degree of colocalization with mStau was revealed by confocal microscopy. Immunofluorescence for mStau $(a)$ reveals selective dendritic labeling (arrows). SYTO14 labeling of the same cell $(b)$ additionally stains axons (arrowheads). Because axons do not contain ribosomes or mRNAs, the labeling most likely reflects mitochondria (Knowles et al., 1996). At higher magnification, mStau $(c)$ and SYTO14 label $(d)$ precisely colocalize in many, but not all, clusters in the dendrites. Arrowheads indicate mStau in SYTO14-labeled RNPs; arrows point to SYTO14 clusters that do not contain mStau. These represent either free mRNA or mitochondria. The average diameter of a CA1 pyramidal neuron is between 8 and $12 \mu \mathrm{m}$. Asterisks mark the branching point of a distal dendrite.

transported to the synapse, most presumably after plastic events (Martin et al., 1997).

What mechanism(s) does neurons use to target mRNAs to dendrites? Putting together the data mentioned above and our own results, it appears logical that the mammalian homolog of mStau is involved in the dendritic transport of mRNAs. This is further substantiated by the fact that mStau has been shown to interact with high affinity with double-stranded RNA, such as the 3'-UTR of bicoid mRNA, and that mStau is specifically associated with polysomes in mammalian cells (Marión et al., 1999). It is tempting to speculate that mStau, by binding to $3^{\prime}$-UTRs of dendritically targeted mRNAs, recruits them into RNPs, which in turn will be moved along MTs into dendrites to the synapses in which they eventually will be translated after synaptic activation. 
These results will now allow the unraveling of the precise role of mStau in the polarized transport of mRNAs in neurons and will pave the way for the future analysis of both the cis-acting transport signals in these mRNAs and its recognition by trans-acting factors, such as the double-stranded RNA-binding protein Staufen. We hope that the characterization of mammalian mStau in neurons opens a new perspective in molecular neurobiology regarding mRNA dendritic targeting and its role in synaptic plasticity.

\section{REFERENCES}

Ainger K, Avossa D, Morgan F, Hill SJ, Barry C, Barbarese E, Carson JH (1993) Transport and localization of exogenous myelin basic protein mRNA microinjected into oligodendrocytes. J Cell Biol 123:431-441.

Ainger K, Avossa D, Diana AS, Barry C, Barbarese E, Carson JH (1997) Transport and localization elements in myelin basic protein mRNA. J Cell Biol 138:1077-1087.

Binder LI, Frankfurter A, Rebhun LI (1985) The distribution of tau in the mammalian central nervous system. J Cell Biol 101:1371-1378.

Bradke F, Dotti CG (1997) Neuronal polarity: vectorial cytoplasmic flow precedes axon formation. Neuron 19:1175-1186.

Breitwieser W, Markussen FH, Horstmann H, Ephrussi A (1996) Oskar protein interaction with Vasa represents an essential step in polar granule assembly. Genes Dev 10:2179-2188.

Broadus J, Westerfield M, Doe CQ (1998) Staufen-dependent localization of prospero mRNA contributes to neuroblast daughter-cell fate. Nature 391:792-795.

Cáceres A, Banker G, Steward O, Binder L, Payne M (1984) Immunocytochemical localization of tubulin and MAP2 during the development of hippocampal neurons in culture. Dev Brain Res 13:314-318.

Campos-Ortega JA (1997) Asymmetric division: intricacies of neuroblast division. Curr Biol 7:R726-R728.

Craig AM, Banker G (1994) Neuronal polarity. Annu Rev Neurosci 17:267-310.

De Hoop MJ, Huber LA, Stenmark H, Williamson E, Zerial M, Parton RG, Dotti CG (1994) The involvement of the small GTP-binding protein Rab5a in neuronal endocytosis. Neuron 13:11-22.

De Hoop MJ, Meyn L, Dotti CG (1998) Culturing hippocampal neurons and astrocytes from fetal rodent brain. In: Cell biology: a laboratory handbook, Ed 2 (Celis JE, ed), pp 154-163. San Diego: Academic.

Deshler JO, Highett MI, Schnapp BJ (1997) Localization of Xenopus Vg1 mRNA by Vera protein and the endoplasmic reticulum. Science 276:1128-1131.

Dotti CG, Sullivan CA, Banker GA (1988) The establishment of polarity by hippocampal neurons in culture. J Neurosci 8:1454-1468.

Ferrandon D, Elphick L, Nüsslein-Volhard C, St. Johnston D (1994) Staufen protein associates with the 3-UTR of bicoid mRNA to form particles that move in a microtubule-dependent manner. Cell 79:1221-1231.

Goslin K, Banker G (1991) Rat hippocampal neurons in low-density culture. In: Culturing nerve cells (Banker G, Goslin K, eds), pp 251281. Cambridge, MA: MIT.

Hill MA, Gunning P (1993) $\beta$ and $\gamma$ actin mRNAs are differentially located within myoblasts. J Cell Biol 122:825-832.
Hortsch M, Avossa D, Meyer DI (1986) Characterization of secretory protein translocation: ribosome-membrane interaction in endoplasmic reticulum. J Cell Biol 103:241-253.

Kislauskis EH, Li Z, Singer RH, Taneja KL (1993) Isoform-specific 3' untranslated sequences sort $\alpha$-cardiac and $\beta$-cytoplasmic actin messenger RNAs to different cytoplasmic compartments. J Cell Biol 123:165-173.

Knowles RB, Sabry JH, Martone ME, Deerinck TJ, Ellisman MH, Bassell GJ, Kosik KS (1996) Translocation of RNA granules in living neurons. J Neurosci 16:7812-7820.

Lawrence JB, Singer RH (1986) Intracellular localization of messenger RNAs for cytoskeletal proteins. Cell 45:407-415.

Li P, Yang X, Wasser M, Cai Y, Chia W (1997) Inscuteable and staufen mediate asymmetric localization and segregation of prospero RNA during Drosophila neuroblast cell division. Cell 90:437-447.

Lucio JP, Brake B, Banting G, Howell KE, Braghetta P, Stanley KK (1990) Identification, sequencing and expression of an integral membrane protein of the trans-Golgi network (TGN38). Biochem J 270:97-102.

Marión RM, Fortes P, Beloso A, Dotti CG, Ortín J (1999) The human homologue of Staufen protein localizes to the polysomes of the rough endoplasmic reticulum. Mol Cell Biol, in press.

Martin KC, Casadio A, Zhu H, E Y, Rose JC, Chen M, Bailey CH, Kandel ER (1997) Synapse-specific, long-term facilitation of Aplysia sensory to motor synapses: a function for local protein synthesis in memory storage. Cell 91:927-938.

Parton RG, Simons K, Dotti CG (1992) Axonal and dendritic endocytic pathways in cultured neurons. J Cell Biol 119:123-137.

Rosa P, Hille A, Lee RWH, Zanini A, De Camilli P, Huttner WB (1985) Secretogranins I and II: two tyrosine-sulfated secretory proteins common to a variety of cells secreting peptides by the regulated pathway. J Cell Biol 101:1999-2011.

St. Johnston D (1995) The intracellular localization of mRNAs. Cell 81:161-170.

Steward O (1997) mRNA localization in neurons: a multipurpose mechanism? Neuron 18:9-12.

Steward O, Levy WB (1982) Preferential localization of polyribosomes under the base of dendritic spines in granule cells of the dentate gyrus. J Neurosci 2:284-291.

van Lookeren Campagne M, Dotti CG, Verkleij AJ, Gispen WH, Oestreicher AB (1992) Redistribution of B-50/growth-associated protein 43 during differentiation and maturation of rat hippocampal neurons in vitro. Neuroscience 51:601-619.

Verkade P, Schrama LH, Verkleij AJ, Gispen WH, Oestreicher AB (1997) Ultrastructural co-localization of calmodulin and B-50/growthassociated protein- 43 at the plasma membrane of proximal unmyelinated axon shafts studied in the model of the regenerating rat sciatic nerve. Neuroscience 79:1207-1218.

Wessel D, Flügge UI (1984) A method for quantitative recovery of protein in dilute solution in the presence of detergents and lipids. Anal Biochem 138:141-143.

Wickham L, Duchaîne T, Luo M, Nabi IR, Des Groseillers L (1999) Mammalian Staufen is a double-stranded RNA- and tubulin-binding protein which localizes to the rough endoplasmic reticulum. Mol Cell Biol, in press. 